\title{
El síndrome de Garp
}

\author{
Manuel González de la Aleja Barberán \\ Universidad de Salamanca
}

\begin{abstract}
The American Novel has been trying since the 1960s to find the way to shape and understand the new reality born in those years. The crisis, we have heard so much about, seems to have its roots in the confusing and paranoid times, all the world, and particularly the United States, are living. Many books and literary trends have tried to explain the new consciousness, the new atmosphere. In this essay, I deal with two books that, in my point of view, have been able to capture in a clear and honest way some of the aspects of a reality that seems more unreal everyday.
\end{abstract}

Cuando se acerca el final de la dêcada de los ochenta parece que todavía somos incapaces de establecer unas coordenadas que definan de forma válida el espíritu anímico, cultural y literario de un período histórico que desde finales de los años sesenta se muestra particularmente confuso e ilógico. En Estados Unidos esta impresión se agudiza de forma característica dada la fuerte personalidad de las decadas anteriores: los años cincuenta y sesenta.

El presidente Ronald Reagan ha insistido con frecuencia a lo largo de todo su mandato en su deseo de recuperar el espíritu de los años cincuenta. Unos años, según él, añorados por una gran parte de la población norteamericana. Evidentemente, la Segunda Guerra Mundial solucionó los problemas económicos y de identidad de un país que se convirtió por fin en la mayor potencia del mundo. Una televisión, un coche, una piscina o una casa en la playa representaban realidades al alcance de todo americano trabajador y temeroso de dios. La guerra fría que ahora se quiere dar por finalizada proporcionaba a una nación compleja y heterodoxa un nexo de unión que, además, reafirmaba sus propias creencias y forma de vida; estaba muy claro quienes eran los buenos y los malos de esa peculiar e híbrida historia.

La década de los sesenta estalló en las mismas narices de esos americanos trabajadores y temerosos de dios de una forma brutal e inesperada. Por momentos, todo pareció tambalearse, incluso principios tan sagrados como la familia y la realidad del Sueño 
Americano. Para otros sectores de la población fueron años de esperanza donde las injusticias latentes en epocas anteriores parecían encontrar un camino de solución. Sus propios sueños y fantasías podían materializarse a través de los ideales y los hechos de figuras emblemáticas como los Kennedy o el reverendo Martin Luther King. Pero, curiosamente, de los años sesenta no ha quedado la materialización de un sueño, sino la realidad de una pesadilla; lo que James Baldwin ha llamado "the utterly intolerable nightmare of the American Dream."1 El asesinato de Robert Kennedy pudo ser, como opina Jack Newfield, el carpetazo definitivo a la esperanza de un mundo mejor:

We felt, by the time we reached thirty, that we had already glimpsed the most compassionate leaders our nation could produce, and they had all been assassinated. And from this time forward, things would get worse; our best political leaders were part of memory now, no hope.

The stone was at the bottom of the hill and we were alone. ${ }^{2}$

Y no sólo se puede entender la desaparición de estas figuras como hechos que cambiaron objetivamente el rumbo histórico del país: sus consecuencias van todavía más allá. Los años sesenta sorprenden por la violencia que desataron y por la incongruencia del desenlace de los pequeños episodios que los componen. Hay en las muertes de sus políticos, en el suicidio de Marilyn, en el asesinato de Sharon Tate y en Vietnam un patrón que no obedece a reglas lógicas o racionales. La muerte y la violencia gratuita se configuran como las pautas que definen la esencia de un país que nació como verdadero paraiso en la tierra. El desencanto de tantas esperanzas frustradas y el terror al nuevo brote de violencia demencial y desmedida van dando forma a unas generaciones incapaces de comprender su realidad o su destino:

And I wondered what terrible venality, what poverty of the spirit, greed, witlesness, what lust for dissolution made my country so violent and ugly and depraved and smug. For it was to have been such a different place. That is what we loved about it. Its former promise. But then I was going to be so different, too. I was going to try to make the world a better place to live. Excuse the dated sentiment, but I was going to try to make it a better place. ${ }^{3}$

Es en estos momentos en los que la figura del escritor sufre más que nadie la banalidad de los tiempos. Es él, más que el político o el asistente social, el que siempre lleva en su fuero interno la esperanza, concebida como deber sagrado, de ser él quien encuentre el camino para hacer de la realidad un sistema comprensible, para conferirle un cierto valor espiritual y moral. Es por esto que él padece como nadie el que no exista patrón posible sobre el que construir un mundo paralelo que explique a la humanidad lo que es o lo que podría ser. La literatura norteamericana a partir de los años sesenta tiene como máxima ineludible las palabras de Philip Roth cuando en su ensayo "Writing American Fiction" afirma:

... the American writer in the middle of the 20 th century has his hands full in trying to understand, and then describe, and then make credible much of the American reality. It 
stupifies, it sickens, it infuriates, and finally it is even a kind of embarrassment to one's own meager imagination. ${ }^{4}$

La periodista y novelista Joan Didion representa a la perfección los efectos traumáticos de la lucha cotidiana del escritor con la realidad violenta y absurda que representa la materia prima de su trabajo. Su artículo "The White Album" es un esfuerzo baldío para ofrecer un retrato coherente de finales de los años sesenta:

We tell ourselves stories in order to live ... We look for the sermon in the suicide, for the social or moral lesson in the murder of five. We interpret what we see, select the most workable of the multiple choices. We live entirely, especially if we are writers, by the imposition of a narrative line upon disparate images. ...5

Y este esfuerzo se lleva a cabo, precisamente, en un momento histórico en el que cualquier intento de comprensión estaba abocado al fracaso:

I was supposed to have a script, and had mislaid it. I was supposed to hear cues, and no longer did. I was meant to know the plot, but all I knew was what I saw: flash pictures in variable sequences, images with no 'meaning' beyond their temporary arrangement, not a movie but a cutting-room experience. ${ }^{6}$

La escritora terminó internada en una clínca mental debido a la incapacidad de su ego para relacionarse con el mundo real y soportar las tensiones normales de una sociedad moderna. En términos psiquiátricos esta peculiar enfermedad recibe el nombre de "The Rosschach Record." En términos literarios podríamos llamarla "El síndrome de Garp" o "El síndrome de Macon." Y su cuadro clínico viene perfectamente esbozado en las novelas The World According to Garp (1976) de John Irving y The Accidental Tourist (1985) de Anne Tyler.

El trabajo de John Irving ha sido con frecuencia tachado de "excesivo." Hay, efectivamente, en todas sus novelas una tendencia "efectista" que agrede sin clemencia el estomago de sus lectores. Asesinatos, violaciones, muertes violentas y absurdas jalonan las páginas de su obra atentando con frecuencia contra la coherencia de un mundo que se nos quiere presentar cercano y cotidiano. Irving se defiende de estas acusaciones insistiendo de manera indirecta sobre el legado que los años setenta recibieron de los "exceso" o los "efectismos" de la década anterior":

People who take exception to the so-called excesses in my work can point out the fact that there's an awful lot of screwing, rape, violence, and other stuff in it that is usually associated with cheap cinema and fiction. I maintain that the stuff of cheap-thrill fiction or film is at our throats all the time. And we all fear it-that's why, if we have the means, we take a cab at night instead of the subway and send our children to private schools so that they won't have to go to public schools. People have to be blind to say that certain things are taboo to write about. ${ }^{9}$

El protagonista de The Accidental Tourist de Anne Tyler también se muestra marcado por los efectos de ese momento histórico. Cuando es consciente de la diferencia de edad 
entre él y su amante, toma precisamente como punto de referencia momentos tan significativos como Vietnam o el asesinato de Kennedy.

El mundo al que la novela se enfrenta hoy en día dejó hace mucho tiempo de seguir un patrón predeterminado que hiciese posible su comprensión. La realidad, "the real and unreasonable world," "10 según Irving y según sus personajes, se asemeja más a "an x-rated soap-opera" 11 "what Christ cooked up in the bathroom." 12 La vida es algo que no se celebra o disfruta, sino más bien algo extraño y lleno de peligros que acechan en cada recodo del camino. Hay una escena en The Water-Method Man que explica de manera sucinta pero brillante toda esta concepción vital. El protagonista de esta obra, como luego ocurrirá a Garp, vive obsesionado por la seguridad de los suyos. Quiere protegerlos a toda costa no sólo de las desgracias posibles que él imagina constantemente, sino también de todos aquellos aspectos negativos que el desearía desechar de un mundo ideal que continuamente construye en su imaginación. Sus esfuerzos se ven, no obstante, una y otra vez superados: incluso visitando el zoo con su hijo, la muerte, el lado oscuro y terrible del mundo que a él tanto le asusta se presenta en la forma de un pato muerto que cae justo a los pies del niño: "the irony of death descending on the Iowa city zoo."13 Esta angustia también termina por desbordar el muro de contención que Macon, el protagonista de The Accidental Tourist, ha intentado construir a su alrededor; cuando cree que ha logrado cierta inmunidad a los imprevistos de su existencia, su relación con un niño al borde siempre de la extinción le devuelve sus antiguas pesadillas:

Those cool little fingers were so distinct, so particular, so full of character. Macon tightened his grip and felt a pleasant kind of sorrow sweeping through him. Oh, his life had regained all its old perils. He was forced to worry once again about nuclear war and the future of the planet. He often had the same secret, guilty thought that had come to him after Ethan was born: From this time on I can never be completely happy. ${ }^{14}$

En The World according to Garp hay asesinatos, accidentes de coche y avión, violaciones brutales, personajes que pierden alguna parte de su cuerpo de forma absurda y trágica. The Accidental Tourist se articula alrededor del asesinato gratuito de un chaval de doce años e incluye otra muerte de una persona joven en accidente de coche. Tal vez en algún momento podamos caer en la tentación de criticar la forma artificial con la que los autores de ambas novelas llevan a sus personajes a situaciones límite. Pero me parece que hoy en día ya no es tan fácil hablar de situaciones límite. La muerte de Ethan en la obra de Ann Tyler palidece ante los asesinatos que leemos en la prensa o vemos en la televisión. Que un fanático dispare en una hamburgersería contra un niño de doce años en el trascurso de un atraco parece que puede obedecer más a la lógica que el hecho real de que un muchacho mate, sin ningún motivo aparente, a catorce chicas y luego se pegue un tiro en la boca. La muerte de Walt en el absurdo accidente de tráfico de la obra de Irving nos es familiar a todos, porque todos hemos vivido ese mismo accidente de una manera u otra. Los asesinatos de Garp o Jenny recuerdan otros muchos, como, por mencionar un ejemplo reciente, el de John Lennon. Lo llamativo de los "excesos" de estas novelas es que no lo son tales y forman ya parte de la vida cotidiana de este nuevo mundo en el que estamos aprendiendo a sobrevivir, pero que cada vez entendemos menos: 
"Macon," she said, "ever since Ethan died I've had to admit that people are basically bad. Evil, Macon. So evil they would take a twelve-year-old and shoot him through the skull for no reason. 1 read a paper now and I despair; I've given up watching the news on TV. There's so much wickedness, children setting other children on fire and grown men throwing babies out second-story windows, rape and torture and terrorism, old people beaten and robbed, men in our very own government willing to blow up the world, indifference and greed and instant anger on every street corner. I look at my students and they're so ordinary, but they're exactly like the boy who killed Ethan. If it hadn't said beneath that boy's picture what he'd been arrested for. wouldn't you think he was just anyone? someone who'd made the basketball team or won a college scholarship? You can't believe in a soul...."15

"The World is too strong" 16 es el principio que rige la vida de Bogus, Garp o Macon. Estos hombres, sobre los que en principio recae la responsabilidad familiar, viven angustiados por dicha responsabilidad. El peligro latente que acecha incansable a sus seres queridos ahoga constantemente sus intentos de ser felices. La vida para ellos dista mucho de ser una celebración, es más bien algo precario, frágil, siempre a punto de romperse en pedazos. El "Under Toad" que Garp imagina agazapado y dispuesto a saltar sobre sus hijos, su esposa o su madre es una realidad que se muestra inflexible cuando menos se la espera: “'Death, it seems,' Garp wrote, 'does not like to wait until we are prepared for it. Death is indulgent and enjoys, when it can, a flair for the dramatic." ${ }^{17} \mathrm{El}$ mundo de estos hombres viene regido por la espera del timbrazo del telefono en medio de la noche:

The telephone, that old cry of alarm-a warrior stabbed on guard duty, screaming his shock - startled the pension where they lived and brought the trembling landlady like a gohst to their rooms. ${ }^{18}$

O por el sentimiento de culpa por no haber podido evitar la tragedia:

He thought Ethan was riding a plaster camel and calling, "Catch me!" and falling. But Macon couldn't get there in time and when he reached his arms out. Ethan was gone. ${ }^{19}$

Precisamente, este sentimiento de culpa, esta incapacidad para aceptar la realidad es la que bloquea las posiblidades de comunicación de estos personajes con los seres que le rodean. Una situación que cobra especial dimensión cuando es retratada precisamente en una novela; un medio cuyo fin último es comunicar algo al lector, encontrar un patrón común de búsqueda que pueda hacer más llevadero el caos en el que todos nos vemos sumidos. La resolución de esta paradoja se muestra como la columna vertebral y la principal virtud de ambas obras.

The World according to Garp,,$^{20}$ como el mismo título indica, pretende recrear un mundo propio que corresponde a la peculiar visión que Garp tiene de la realidad. Muchos de los hechos que tienen lugar en el libro materializan de una forma u otra la peculiar metafísica del personaje central, creando un curioso vínculo entre el mundo exterior a Garp y sus pensamientos más íntimos plasmados continuamente en la obra. No obstante, la narración en tercera persona a cargo de un narrador omnisciente, permite al autor ofrecer 
otros "mundos según" otros personajes. Estos mundos se interrelacionan entre ellos y con el mundo según el protagonista de manera complicada y precaria, y, con frecuencia, el resultado de esta relación es la violencia, la tragedia o simplemente el desamor. The Accidental Tourist está escrito en tercera persona, pero una tercera persona que recoge siempre el punto de vista de Macon. La riqueza de personajes recreados desde el punto de vista de Garp o materialmente inventados por él, dan paso aquí a una galería de personajes que existen en función de uno principal. Percibimos a aquellos según Macon los siente o, como es en la mayoría de los casos, según no quiere sentirlos. Adoptando el punto de vista de un ser parapetado tras su dolor la autora puede recrear de manera sutil la angustiosa soledad del ser humano. Partiendo de esta misma premisa, ambos autores crearán su propia serie de imágenes o situaciones para ilustrarla.

Irving materializa la idea de la inadaptación y la incomuncación a través, principalmente, de las incapacidades físicas de algunos de los personajes, del sexo y el trabajo de Garp como escritor.

La novela de Irving ofrece una amplia galería de personajes con alguna mutilación o tara física. Evidentemente, no hay un sádico afán de burla en ello, sino precisamente la materialización de la idea de que el ser humano se encuentra con frecuencia impedido en su captación del mundo y su relación con los demás. La perdida del ojo y el brazo de Duncan muestran lo doloroso de las muertes trágicas de su hermano, su abuela y su padre, y su lucha por adaptarse a ellas. Las dificultades en el habla de Alice Fletcher y Tinch hacen más patético su deseo de salir de su soledad y comunicarse con los demás. Las “Ellen Jamesians" ilustran de forma trágica la necesidad de amor convertida, con frecuencia, en furia fanática: su autoamputación, absurda y gratuita, es un acto de violencia desesperada con el que intentan decirle a los demás que difílcilmente soportan la violencia a la que, como mujeres, se ven frecuentemente sometidas; incluso el propio Garp terminara por comprender este gesto desesperado poco antes de ser asesinado por una de ellas.

El sexo supone una preocupación constante en la novelística de Irving; a él ha dedicado incluso toda una novela, The 158-Pound Marriage. El sexo, la lujuria que llamaría Jenny Fields, aparece en su obra más como una barrera entre los seres humanos antes que como el puente capaz de unirlos. ${ }^{21}$ "Human sexuality makes farcical our most serious intentions," 22 afirma. El adulterio se convierte en la amenaza real en la convivencia de los personajes; la muerte de Walt viene causada por la infidelidad de Helen, pero, también, porque Garp no quiso hacer el amor con ella la noche que más lo necesitaba:

Garp went upstairs. When she came up to him, he was already asleep, which made her despair. If he'd had her on his mind at all, how could he have fallen asleep? But, actually, he'd had so much on his mind, he'd been confused; he had fallen asleep because he was bewildered. If he'd been able to focus his feelings on any one thing, he'd still have been awake when she came upstairs. They might have saved a lot of things, then. ${ }^{23}$

La violación se ofrece como la culminación más indeseable de un acto que en vez de crear amor o entendimiento, crea dolor y muerte. La bestial violación de la novela escrita por 
Garp representa la situación límite de unos seres humanos que no son capaces de canalizar de forma lógica sus sentimientos. La violación de Ellen James priva a la niña precisamente del que será su mayor deseo, poder hablar con sus semejantes.

Pero es sobre todo la condición de novelista de Garp la que cuestiona las posibilidades de comunicación del ser humano en este presente caótico. Hay en todas las obras de Irving un deseo por parte de sus personajes de contruir un refugio capaz de aislarlos del mundo real: en Setting Free the Beers, para Siggy ese refugio está en su "autobiografía" y en su motocicleta; en The 158-pound Marriage, para uno de los personajes la lucha grecoromana constituye el lugar ideal donde olvidar las zancadillas de la realidad cotidiana; ${ }^{24}$ Bogus, en The Water-Method Man, es un soñador capaz de perderse en todo momento en sus fantasías de héroes, princesas y enormes ballenas blancas. El sueño de Garp por construir una especie de fuerte capaz de proteger a Helen y a sus hijos de todos los peligros del mundo exterior encuentra su réplica en su trabajo como novelista:

... the act of starting a novel felt like: "It's like trying to make the dead come alive," he said. "No, no, that's not right-it's more like trying to keep everyone alive, forever. Even the ones who must die in the end. They are the most important to keep alive."2s

Garp llega a sostener que lo que un hombre inventa es más importante que la realidad: "What was 'going on,' in Garp's opinion, was never as important as what he was making up-what he was working on." ${ }^{26}$ Pero, fiel a las palabras de Roth, este novelista llegará un momento en que no podrá combatir con la realidad y verá como su imaginación es paulatinamente aniquilada por los sucesos presentes que le asedian. Garp escribe su obra más brillante en la juventud, y más tarde la muerte trágica de sus seres queridos le impedirán escapar de sí mismo y poder crear. La literatura se muestra ineficaz como refugio, pero, además, en vez de unir a Garp con sus semejantes provocará el odio que finalmente le destruirá.

Aunque los dos autores partan de ideas parecidas y lleguen a conclusiones similares, es evidente el contraste entre el tipo de narrativa que nos presenta John Irving y el que ofrece Ann Tyler. Un contraste evidenciado por el tipo de protagonista que eligen para sus respectivas novelas. Frente al carácter paranoico y creativo de Garp, Tyler nos propone un personaje que trata de anular toda su capacidad de sentir para poder evitar así el dolor. Si el primero escribe novelas y trata de crear un mundo acorde a sus ideas, Macon Leary pretende la huida, pasar por un mundo caótico de acuerdo a un "sistema," a un "método." La imposibilidad de comunicación de los distintos mundos en Garp, viene ilustrada en The Accidental Tourist de forma mucho más sutil y sobria. Así, el retrato que Tyler nos da de la familia de Macon significa con un humor triste y tierno la incapacidad de estos seres adultos para relacionarse con las más pequeñas contingencias y peligros del mundo real: los Leary nunca contestan al telefono, se pierden incluso cuando van de su casa a la ferretería y pasan las tardes jugando a un juego de cartas que sólo ellos entienden y que parece vetado a cualquier intruso. El cuadro en la pared de la casa familiar refleja el aislamiento de estos seres y también el vacío de unas vidas que no se quieren o no se pueden vivir: 
In the portrait on the end wall, the Leary children gazed out with their veiled eyes. It occurred to Macon that they were sitting in much the same positions here this evening: Charles and Porter on either side of him. Rose perched in the foreground. Was there any real change? He felt a jolt of something very close to panic. Here he still was! The same as ever! What have I gone and done? he wondered, and he swallowed thickly and looked at his own empty hands. ${ }^{27}$

Pero, evidentemente, la idea que mejor ilustra todo este concepto de la soledad y la incomunicación es la afortunada imagen de las guías de viajes que Macon escribe y que dan título a la novela:

He covered only the cities in these guides, for people taking business trips flew into cities and out again and didn't see the countryside at all. They didn't see the cities, for that matter. Their concerned was how to pretend they had never left home ... Other travelers hoped to discover distinctive local wines; Macon's readers searched for pasteurized and homogenized milk. ${ }^{28}$

El afán de Macon como viajero es el mismo que su afán como ser humano: poder pasar por la vida sin ser contaminado por sus tragedias e incomodidades. El libro en el que se sumerge en el avión o el tren para evitar tener que hablar con su compañero de viaje equivale a su ferviente deseo de evitar tener que soportar la dolorosa relación con sus semejantes. Incluso con la muerte de su hijo renuncia a recibir la compasión o el cariño de las personas que le rodean. Precisamente, un vecino deseoso de tenderle la mano, es el que mejor describe su actitud ante la vida y la causa por la que ha perdido a su mujer:

Just look at the way you walk! The way you, like, lunge, lope on down the street with your head running clear in front of your body. If a fellow wants to stop you and, I don't know, offer his condolences, he'd be liable to get plowed down. Now, I know you care, and you know you care, but how does it look to the others? I ask you! No wonder she up and left. ${ }^{29}$

La narración de la novela se ajusta perfectamente a la actitud y sentimientos de Macon. El “mundo según Macon" fluye ante los ojos del lector de manera casi imperceptible, con la intromisión mínima del narrador; las transformaciones que se van produciendo surgen imprevistas, casi desapercibidas tanto para el personaje como para el lector. ${ }^{30}$ Macon pasa de un estadio a otro arrastrado por su propia soledad, por su incapacidad para ofrecer amor o recibirlo sin temor, por la imposibilidad de controlar los sucesos en los que se ve inmerso.

Todo lo dicho anteriormente da una imagen un tanto equívoca de las obras comentadas. Pese al punto de vista especialmente pesimista del que parten tanto Tyler como Irving, ambos escritores, probablemente precisamente por serlo, parecen sentir la obligación de ofrecer una vía de escape a unos personajes marcados por la tragedia, de dar con su obra una posibilidad a la esperanza tanto en su ficción como en la realidad que atañe a sus lectores. Son las suyas novelas que emanan un optimismo que, aún siendo muchas veces doloroso, brinda al lector un punto de referencia válido para comprender la 
incomprensible realidad. Los mecanismos que utilizan para conseguirlo son distintos pero apuntan por igual al alma misma de la novela.

The World according to Garp sorprende por los intensos contrastes que la comedia y la tragedia están continuamente generando: "In the world according to Garp, an evening could be hilarious and the next morning could be murderous." ${ }^{31} \mathrm{La}$ violencia que preside la obra viene siempre matizada por un tono absurdo o irónico que desconcierta una y otra vez al lector: la muerte de Walt "causada" por el juego inocente de Garp y la felatio que Helen le está haciendo a su amante, que resulta castrado en el incidente; el entierro de Jenny al que su hijo tiene que acudir disfrazado de mujer en una desconcertante escena típica de la comedia norteamericana de los años cuarenta y cincuenta; la muerte de Duncan, asfixiado por una aceituna en pleno ataque de risa; la propia imagen del "Under Toad," resultado de una tierna y profética confusión de un niño que penso que lo peligroso del mar no era la resaca ("Under Tow"), sino un sapo verde y maligno; y, por su puesto, la muerte de Garp:

It was a death, Wolf thought, which in its random, stupid, and unnecessary qualities-comic and ugly and bizarre-underlined everything Garp had ever written about how the world works. ${ }^{32}$

Garp no puede evitar pensar que muchos de los problemas de los seres humanos son divertidos, pese a que nos neguemos a aceptarlo. Todas las acciones de los seres humanos resultan ridículas y apenas ocultan su lado burlesco. Violencia y sexo, son presentados como situaciones extremas a las que los personajes son sometidos en un continuo tono de farsa.

Pero, pese a que pueda parecer que Garp es un libro cruel o ciertamente cínico, el humor que preside estas tragedias es el que precisamente las hace más soportables y establece un vínculo definitivo entre las personas que las sufren y entre el libro y su lector. La risa no sirve para menospreciar el dolor, sino para compartirlo; establece una corriente de simpatía entre los seres abatidos por la desgracia: "Garp believed that laughter was related to sympathy, which we were always needing more of."33

Es decir, el humor que emanan en general de las novelas de Irving no nace de la sátira - la parodia, sino que está íntimamente ligado a la capacidad de su obra por humanizar, por sentimentalizar, por atraer al lector hacia lo incomprensible y lo grotesco. Sus novelas parten de la base de que el ser humnano no puede ni comprender la realidad absurda que le rodea, ni tampoco establecer una comunicación válida con sus semejantes. The World according to Garp cuestiona incluso la validez de la novela como un camino para la comprensión de y entre los hombres; Garp utiliza la literatura más para huir que para analizar o entender. Pero, paradójicamente, todas sus obras presentan a personajes con una enorme capacidad para sentir y para amar. Probablemente no se comprendan claramente unos a otros, pero son capaces de sentir como suyas las desgracias ajenas. La sensibilidad a flor de piel de Garp suaviza y da significado a las situaciones trágicamente cómicas en las que se ve envuelto. Las obras de Irving pretenden, sobre todo, hacer comprender al lector que todos tenemos derecho a cabalgar como buenamente podamos sobre nuestro "hobby-horse" particular. Todos los seres humanos tienen el derecho a imaginar, recordar 
o inventar una dignidad válida para sus vidas. Es legítimo que el viejo Bodger crea firmemente que cogió a Garp cuando cayó del tejado en vez de a una paloma. Y es legítimo que un gigantesco jugador de Futbol Americano cree de sí mismo una frágil y tierna mujer llamada Roberta. Así, Garp sólo podrá escribir de nuevo $0^{34}$ cuando sea capaz de admitir la existencia de las "Ellen Jamesians" y pueda sentir cariño hacia la esposa del hombre que mató a su madre. Probablemente, esos mundos peculiares y herméticos de cada uno de los personajes los hagan, como a los seres humanos de carne y hueso, totalmente incomprensibles, pero en el momento que entendemos su intento por huir de una realidad que es para ellos tan absurda como para nosotros, haremos de sus causas, nuestras causas y lloraremos sus perdidadas como nuestras perdidadas. Recordemos que sólo los necios ven a Don Quijote como un ser ridículo.

Anne Tyler, minuciosamente unida al pensamiento y ánimo de su personaje, ofrece a través de él esa puerta a la esperanza que su condición de escritora le exige. Con el, la autora muestra la imposibilidad de renunciar de forma definitiva a la existencia y la necesidad de hacer de los semejantes nuestro punto de referencia obligado para poder sobrevivir, "He began to think that who you are when you're with somebody may matter more than whether you love her." 35 La experiencia vital de Macon así lo demuestra.

Lo único que conecta a Macon con su pasado y con el exterior es Edward, el perro de su hijo, cuyo carácter empieza a modificarse tras el asesinato de su dueño, y del que no puede desprenderse pese a los consejos de familiares y conocidos. Y el perro le lleva a Muriel, una mujer extraña, marcada también dolorosamente por la vida, pero con un afán desmedido por luchar y ser feliz. Macon empieza su vida con Muriel y Alexander de forma casi involunataria, la inercia le lleva a una casa donde empezará a sentirse vivo de nuevo pero donde se verá una vez más amenazado por el dolor y la tragedia. Si Ethan, aún siendo un muchacho fuerte y sano, lleno la vida de su padre de miedos y angustias, parece absurdo que Macon vaya a vivir con Alexander, un niño al que puede matar la picadura de una abeja. Pero el "turista accidental" piensa que esa mujer y su niño no le pertenecen de la misma forma que lo hizo su familia anterior; siempre estará a tiempo de hacer las maletas y seguir su viaje: "He could still draw back from Alexander; he could still give up on him." 36 Es por ello que cuando Muriel insiste en sus demandas de matrimonio; cuando Alexander se convierte en una dolorosa realidad como lo fue Ethan; o cuando Macon se sorprende a sí mismo hablando con sus compaeñeros de avión, de nuevo de forma involuntaria, casi sin querer, vuelve a su casa y a Sarah:

He came to Singleton Street and flicked his signal on but didn't turn. After a while the signal clicked off on its own. He rode on through the city, up Charles Street, into his old neighborhood. Ha parked and cut the engine and sat looking at the house. The downstairs windows were dark. The upstairs windows were softly glowing, he had come home. ${ }^{37}$

No obstante, su contacto con el mundo exterior, con una realidad que no es la que él había creado de forma artificial, lo transformará definitivamente. Macon empieza a ver la humanidad sumida en un intento desesperado por comunicarse con los demás. De la mano de Muriel se imagina a los diminutos seres humanos vistos a la puerta de un hospital por un ser de otro planeta como criaturas siempre deseosas de ayudar a sus semejantes. Es él 
quien intentará convencer a Sarah de que todo no puede ser tan negativo: “. . it's kind of heartening, isn't it? How most human beings do try. How they try to be responsible and kind as they can manage." ${ }^{37}$

El barrio de Muriel en principio retratado como sórdido y peligroso va trasformandose en un lugar acogedor y bullicioso donde todos sus habitantes tratan de dignificar su existencia. Incluso París, una ciudad carente de romanticismo o educación, se convierte en el lugar que ofrece a Macon la llave de una posible felicidad:

The taxi lurched to a halt. A sudden flash of sunlight hit the windshield, and spangles flew across the glass. The spangles were old water spots, or maybe the marking of leaves, but for a moment Macon thought they were something else. They were so bright and festive, for a moment he thought they were confetti. ${ }^{39}$

De nuevo, la tragedia ridícula del ser humano se convierte en la epopeya de unos seres deseosos de amar y ser amados.

En todos los libros que hemos comentado aquí parece que existe un anacrónico contraste entre la crueldad del mundo que representan y la ternura con que contemplan a los seres que los pueblan. Una ternura que emana del empeño de todos ellos por encontrar el lugar que les corresponde en ese mundo absurdo. Los personajes de Irving o Tyler se muestran incapacitados para relacionarse con la realidad pero se ven continuamente atraidos hacia ella. En Irving, la muerte de un pato en el Zoo o el asesinato de la madre obligan a Bogus o a Garp a reconocer que la vida no es una romática leyenda o una "oldfashion novel," sino algo absurdo; pero eso no quita para que la risa y la ternura les haga aceptar como es ese mundo y todo lo que contiene. Bogus reclama para sî la imagen de la ballena blanca que aun dolorida y cansada se niega a claudicar: "Mindful of his scars, his old harpoons and things, Bogus Trumper smiled cautiously at all the good flesh around him. " ${ }^{40}$ Y Garp reconocerá en el momento mismo de su muerte que todos sus miedos son parte indispensable de la vida que intento gozar:

If he could have talked, he would have told Helen not to be frightened of the Under Toad anymore. It surprised him to realize that the Under Toad was no stranger, was not even mysterious; the Under Toad was very familiar-as if he had always known it, as if he had grown up with it. ${ }^{41}$

En la obra de Tyler, Macon deberá asumir que el tiempo pasa, que no puede renunciar al paisaje que le rodea, que tarde o temprano pedirá ayuda o ayudará a su compañero de asiento en el avión, que el dolor forma parte de la exitencia y del fluir de la vida:

The real adventure, he thought, is the flow of time; it's as much adventure as anyone could wish. And if he pictured Ethan part of the flow-in some other place, however unreachable - he believed he might be able to bear it after all. ${ }^{42}$

Es probable que tanto The World according to Garp como The Accidental Tourist no hayan servido para solucionar la eterna crisis de la novela y que tampoco hayan logrado 
convertirse en esa "gran novela americana" que todos añoran. Sus innovaciones técnicas no exiten o pueden resultar un tanto pueriles. Pero, recordemos, que la literatura tiene la obligación de hacer nuesta existencia más soportable. Un buen libro, independientemente de "enriquecer nuestro curtido intelecto," debe recordarnos que no estamos sólos, que exiten otros seres que sufren y ríen como nosotros lo hacemos. Si estos libros son bestsellers, eso no quiere decir que sean despreciables, sino que han logrado captar el espíritu de unos tiempos que a muchos nos dan mucho miedo.

\section{Notas}

1. James Baldwin, The Evidence of Things Not Seen (New York: Holt, 1985), p. 82.

2. Jack Newfield, Robert Kennedy: A Memoir (New York: Dutton, 1969), p. 304.

3. Dotson Rader, I Ain't Marchin' Anymore (New York: David Mckay, 1969), p. 3.

4. Citado por John W. Aldridge, The American Novel and the Way We live Now (Oxford: Oxford UP, 1983), p. 28.

5. Joan Didion, "The White Album," en The White Album (New York: Washington Square Press, 1979), p. 11.

6. Didion, p. 13.

7. The New Yorker dijo de The World according to Garp: "Scores of characters die violently and dozens are melodramatically (and unconvingcingly) maimed."

8. John Irving ha negado alguna vez la influencia en su literatura de los sucesos ocurridos en su país en aquella década, alegando que el se encontraba en Viena por aquel entonces. Yo creo que esta afirmación le sirve para defenderse de las implicaciones políticas que autores como Larry McCaffery han visto en The World according to Garp o The Cider House Rules, pero que no desdice la influencia que la violencia y la inestabilidad de esos hechos provocaron en la mentalidad norteamericana.

9. Tom LeClair and Larry McCaffery, Anything Can Happen (Urbana: University of Illinois Press, 1983), pp. 190 y 191.

10. John Irving, Setting Free the Beers, en 3 by Irving (New York: Random House, 1968), p. 269.

11. John Irving, The World according to Garp (New York: Henry Robbins Books, 1976), p. 338.

12. Irving, Setting Free the Beers, p. 57.

13. Irving, The Water-Method Man, en 3 By Irving, p. 397.

14. Anne Tyler, The Accidental Tourist (Harmonsworth: Penguin Books, 1986), p. 258.

15. Tyler, p. 140.

16. Irving, The Water-Method Man, en 3 By Irving, p. 304.

17. Irving, The World according to Garp, p. 367.

18. Irving, The World according to Garp, p. 345.

19. Tyler, p. 38.

20. Como el propio Irving ha reconocido en alguna ocasión la trilogía que precede a The World according to Garp prepara el camino a una obra definitiva que sería precisamente esta novela de 1976. Es por ello que este estudio toma esta novela y a Garp como principales puntos de referencia. Esto no quita que haga referncias a las obras anteriores, principalmente a The WaterMethod Man y su protagonista Bogus, el precedente más evidente de Garp. 
21. La idea del amor entendido como problema más que como solución se repetirá en The Hotel New Hampshire, donde los dos hermanos tendrán que hacer una y otra vez el amor para que uno de ellos pueda olvidar su incestuosa obsesión y relacionarse de forma válida con el resto del mundo. En The Cider House Rules el sexo aparece como el responsable de pequeñas tragedias cotidianas y niños no deseados que envejecen esperando una madre y un padre que los amen.

22. Irving, The World according to Garp, p. 160.

23. Irving, The World according to Garp, p. 329.

24. Esta imagen se vuelve a repetir con Garp, el cual, al igual que su creador, se convierte en entrenador de este deporte. Así, es en una colchoneta del gimnasio donde Garp hace el amor con Helen por primera vez y también es en una colchoneta donde es asesinado.

25. Irving, The World according to Garp, p. 409.

26. Irving, The World according to Garp, p. 135.

27. Tyler, pp. 80-81.

28. Tyler, p. 12.

29. Tyler, p. 75.

30. Buena muestra de esta sobriedad narrativa es el comienzo del libro. En las tres primeras páginas el lector ve como el matrimonio de Macon se disuelve tras las perdida de Ethan. El capítulo 2 empieza ya con la frase "after his wife left him," p. 8.

31. Irving, The World according to Garp, p. 406.

32. Irving, The World according to Garp, p. 414.

33. Irving, The World according to Garp, p. 166.

34. La nueva novela que Garp prepara tiene precisamente como protagonista a un padre soñador que arrastra alegremente a toda su familia hacia la imposible materialización de sus fantasías. La novela que Irving escribe después de Garp, The Hotel New Hampshire, retoma este mismo tema e incluso logra hacer realidad alguno de los sueños de su personaje.

35. Tyler, p. 317.

36. Tyler, p. 238.

37. Tyler, p. 301. En el siguiente capítulo Macon aparece ya en su vida cotidiana con Sarah. Al igual que con su separación o cuando se va a vivir con Muriel, la autora ofrece los hechos ya consumados, sin ofrecer la explicación o el razonamiento que el propio protagonista se niega a sí mismo.

38. Tyler, p. 350.

39. Tyler, p. 355.

40. Irving, The Water-Method Man, p. 560.

41. Irving, The World according to Garp, pp. 412 y 413.

42. Tyler, p. 354. 